year period. While accounting for surgical BMI and age, quantile regression was used to create expected WL curves (10th, 25th, 50th, 75th, and 90th \%tile) for the 36 month post-operative period. Results: A mobile application (Get-2-Goal) was designed to provide a simple, personalized interface that allows patients to track their WL and compare their WL results to their expected WL curves. Get-2-Goal was made publicly available at no cost on a popular Apps store and is compatible with current smartphone and tablet technology ( $>1000$ downloads to date). Get-2-Goal allows patients to input their personal profile (e.g. age, BMI), review their expected WL, and track their WL post-operatively. Patients have the option of saving a graphic containing their personalized WL curves and e-mailing a tabular form of their WL results to family, friends, and/or care providers. Conclusions: Get-2Goal is a simple tool that may be used by Bariatric Surgery Programs to facilitate electronic patient education. This tool may assist patients in deciding to proceed to WL surgery, and will facilitate early identification of patients who are struggling with WL or weight maintenance. Future modifications of this methodology can be used to personalize other comorbidity outcomes such as diabetes resolution.

Keywords: Patient-centered; Bariatric surgery

$$
\text { doi:10.3121/cmr.2014.1250.ps1-48 }
$$

\section{Embedded Research}

A3-1:

The Just Do It Playbook for Implementation Science

Adam Sharp ${ }^{1}$; Huong Nguyen'; Erin Hahn ${ }^{1}$; Tania Tang${ }^{1}$; Brian Mittman ${ }^{1}$; Michael Kanter ${ }^{1}$; Steve Jacobsen ${ }^{1}$; Michael Gould ${ }^{1}$

\section{${ }^{1}$ Kaiser Permanente Southern California}

Background/Aims: There are over 60 published implementation science frameworks, and despite the advantages of each, none were designed to help clinicians and administrators to standardize and prioritize initiatives, implement programs efficiently, and evaluate with sufficient rigor. Methods: In order to address these limitations, the Care Improvement Research Team (CIRT) at Kaiser Permanente Southern California blended implementation and behavior change frameworks into a structured process to help clinicians, operations leaders and researchers to standardize their approach and accomplish collaborative goals. To develop the "Just Do It Playbook," we performed a pragmatic literature review, compared and synthesized published frameworks, and iteratively refined a set of steps for identifying, diagnosing and correcting gaps in care. Results: The Just Do It Playbook can be simplified into four distinct steps: 1) describe the topic that needs improvement, 2) identify what or who needs to change, 3) develop an implementation strategy to facilitate change, and 4) evaluate the effectiveness of the strategy. The first step helps to prioritize the topic of interest and identifies whether there is a gap between current and best practices. Second, describing what or who needs to improve will enable identification of barriers or facilitators to change. The third step identifies strategies to promote best practices by using established behavior change theories as well as clearly defined and measurable outcomes. The final step evaluates the outcomes, costs and sustainability of the strategy, as well as the need to discontinue efforts, modify and re-evaluate, or disseminate to other settings. Conclusions: In a learning health care system, there are many potential targets for improvement, and many strategies for implementation and evaluation. One way to simplify the complexities that inherently exist in health system improvement is to adopt a standardized approach. As clinicians, administrators and researchers forge meaningful collaborations, a standard process can improve the understanding of competing priorities, define roles and responsibilities, and integrate established scientific frameworks in a way that is efficient and sustainable. The Just Do It Playbook will need to be tested and adapted to individual projects, but will provide a platform to build embedded research into a functional operational standard.

Keywords: Research-operations partnership; Implementation science doi:10.3121/cmr.2014.1250.a3-1
A3-2:

Mindful Inquiry: From an Observer to a Gentle Change Agent in the Prevailing Culture of Do and Tell

Ming Tai-Seale ${ }^{1}$

${ }^{1}$ Palo Alto Medical Foundation Research Institute

Background/Aims: In a culture of do and tell, researchers can use massive amount of EHR data, find statistically significant results, and publish them, often without the need to interact with the people who generated those data through their daily work or their use of health care services. Such observational inquiry can be informative one hand, as identifying areas for improvement requires the identification of gaps in quality of care or other shortcomings in the system. Mindful inquiry, i.e., asking questions in the moment, the here-and-now, on the other hand, has the potential to become a change agent that can facilitate the creation of new knowledge and the dissemination and implementation of best practices. Methods: Appreciative inquiry. Results: Task oriented culture of research values doing more than relating, telling more than asking, thereby leaving room for improvement in our capacity and desire to form and keep relationships with our research subjects or the organization where we do research. The practice of fly-bynight researcher who swoops down, collects data, publishes papers that expose dark sides of the subjects' lived world has caused permanent harm to communities. Embedded researchers need to be mindful about the questions they raise, the methods they employ, and how they disseminate the results. Conclusions: Embedded research is a series of complex, interdependent tasks which requires building positive, trusting relationships with research team and delivery system partners to facilitate good communication. We must be better at asking and do less at telling. Transform our mindset from the researcher knows a lot to the practitioners know a lot. We must be open to adaptive learning, be curious and courageous enough to ask questions to which we do not already know the answer. Good communication is key to building a trusting relationship which requires mindful inquiry, done with humility to build positive relationships with the delivery organization and guided by the wish to be of service to the greater good.

Keywords: Embedded research; Change agent

doi:10.3121/cmr.2014.1250.a3-2

\section{A3-4:}

Implementing a Pragmatic Clinical Trial: Lessons Learned about Embedded Research

Lindsay Kindler'; Carmit McMullen ${ }^{1}$; Ashli Owen-Smith²; Stacey Honda ${ }^{3}$; Alison Firemark ${ }^{1}$; Lynn DeBar ${ }^{1}$

${ }^{1}$ Kaiser Permanente Northwest; ${ }^{2}$ Kaiser Permanente Southeast; ${ }^{3}$ Kaiser Permanente Hawaii

Background/Aims: Pragmatic trials are fostering a paradigm shift to increasingly embed research into healthcare settings. Pragmatic trials test interventions in real world settings representative of those targeted for dissemination to promote robust, sustainable changes within the health care system. Kaiser Permanente's Center for Health Research, is conducting a pragmatic trial to test the effectiveness of teaching pain self-management skills using interdisciplinary teams within primary care in three Kaiser regions. Implementation requires a pragmatic approach to create a working relationship between health system leaders and the research team. We present lessons learned during our preparatory year in which we partnered with health systems to build an embedded research infrastructure for the study. Methods: In accordance with a pragmatic approach, we used the Rapid Assessment Process (RAP) whereby the fieldwork team gathers and analyzes information quickly in the form of journal entries and detailed meeting minutes following stakeholder encounters and regular team meetings to document the emerging collective understanding of stakeholders' expressed concerns and informational needs. This approach allows for iterative feedback from operational stakeholders and accommodates quick trial modifications. Results: Using RAP to systematically identify lessons learned, several guiding principles surfaced for successfully embedding research into a healthcare system. The study foundation must come from a need identified by the health system, not the research team. Study outcomes must have obvious utility to operational leaders and practicing providers. 